\section{Low incidence of EPOR mutations in idiopathic erythrocytosis}

Since the discovery of $J A K 2$ mutations, the etiological diagnosis of erythrocytosis has been greatly facilitated. When positive, the diagnosis of polycythemia vera $(\mathrm{PV})$ is highly probable; when negative, it is necessary to focus on secondary causes, particularly hypoxia due to cardiorespiratory diseases or tumor-related causes. However, the cause of erythrocytosis often remains unknown even after extensive investigation, which leads to the diagnosis of idiopathic erythrocytosis, or primary familial and congenital polycythemia. We report here the low incidence of mutation $(1.1 \%)$ in the erythropoietin receptor $(E P O R)$ gene in a large series of 270 consecutive unrelated cases with primary familial and congenital polycythemia. Three mutations in the EPOR gene were observed, two of which have already been reported as pathogenic (Pro381Glnfs*2 and Ser407*). The third mutation is a new variant (Ser432Alafs*21) that was discovered in one patient from New Caledonia. Interestingly, a different French laboratory found the same variant in three other patients from New Caledonia without known family ties.

Erythrocytosis and polycythemia are common terms referring to the increase of hematocrit $(\mathrm{Ht})$ and hemoglobin $(\mathrm{Hb})$ concentrations. However, to ensure a diagnosis of true erythrocytosis, certain criteria must be met. Testing must reveal a red cell mass (RCM) $>125 \%{ }^{1}$ or $\mathrm{Ht}$ $>56 \%$ in women or $>60 \%$ in men.

Primary erythrocytosis is caused by an intrinsic abnormal proliferation of the erythroid compartment. The most common is PV, the diagnosis of which is well standardized according to the 2016 World Health Organization (WHO) criteria, ${ }^{2}$ which includes the JAK2 mutations, found in $98 \%$ of cases. Erythrocytosis can also result from an acquired abnormality that stimulates erythropoietin (EPO) secretion, resulting in secondary polycythemias. Increased secretion of EPO can arise from hypoxia pathway activation, often observed in heart or lung diseases, and also from ectopic secretion of EPO by tumors. Congenital secondary erythrocytosis results, among others, from hemoglobin with high oxygen affinity due to $\alpha, \beta$ or $\gamma$ globin mutations, or from a very rare mutation in the bisphosphoglycerate mutase (BPGM) coding gene that causes 2,3-BPG levels to decrease. ${ }^{3}$ Yet many cases of polycythemia remain idiopathic because the most common causes are not identified. Since the advent of molecular biology and next-generation sequencing (NGS), the genes involved in the regulation of the hypoxia sensing pathway (PHD2/EGLN1, HIF2A/EPAS1, VHL, EPO) or in proliferation and differentiation of erythroid progenitors JAK2 or the EPO recep- tor gene [EPOR]) are more easily identified and their pathological pathways can be clarified. As recently reviewed, new mutations in the HIF/EPO pathway have been reported in the last few years, underscoring the rapid evolution of current knowledge on the causes of erythrocytosis. ${ }^{4}$ For example, we recently demonstrated that exon skipping and cryptic exon retention were new $V H L$ alterations and revealed a novel complex splicing regulation of the VHL gene.

Over the past 4 years, 270 patients (229 males, 41 females) with idiopathic erythrocytosis have been tested using a dedicated NGS panel of genes involved: (i) in the regulation of the hypoxia pathway (PHD1 [EGLN2], PHD2 [EGLN1], PHD3 [EGLN3], HIF-1A, HIF-2A [EPAS1], HIF-3A,VHL,VHLL); (ii) in proliferation and differentiation of erythroid progenitors (EPO, EPOR, $J A K 2, L N K$ [SH2B3], $C B L$ ); or (iii) in mature cell function (bisphosphoglyceratemutase (BPGM]). In order to restrict our analysis to truly idiopathic polycythemia, patients were selected according to clinical and biological criteria: increased RCM $>125 \%$ (measured using a chromium 51 method) or Ht value $>56 \%$ in women or $>60 \%$ in men were necessary for the confirmation of absolute erythrocytosis. Each patient underwent testing for blood electrolytes, serum EPO, JAK2 mutations (on exon 14 and exon 12), functional respiratory tests, abdominal ultrasound scan, arterial and venous blood gas (in search of a $\mathrm{P} 50<24 \mathrm{mmHg}$ that could be indicative of $\mathrm{Hb}$ with high oxygen affinity) in order to eliminate classical secondary causes. In particular, the measurement of venous $\mathrm{P} 50$ is a rapid, low-cost test that can orientate further screening towards an inexpensive sequencing technique for the $H B B, H B A 1$ and $H B A 2$ genes. ${ }^{6,7}$

Finally, because the detection of variants in the genes with high similarity of sequences such as HBA1 and HBA2 is challenging using NGS, we decided not to include $H B B, H B A 1$ and $H B A 2$ genes in our NGS panel. ${ }^{8}$

Patients were included in the NGS program if their results showed no abnormalities in the criteria cited above. Using the NGS panel, variants considered as pathogen were observed in $62(23 \%)$ patients, mainly in the genes involved in the regulation of the hypoxia pathway. Concerning the EPOR gene, only three different mutations, all in exon 8 , were identified in three of the 270 patients that were tested (Table 1 ).

The first anomaly, a frameshift mutation (c.1142_1143delCC, p.Pro381Glnfs*2), was found in patient \#1: a 52-year old woman. A family history of probable erythrocytosis in her father and brother was reported, but further information was not available.

The second EPOR mutation, a nonsense mutation (c.1220C>A, p.Ser407*), was found in patient \#2, a 14year old girl, who had been diagnosed with idiopathic

Table 1. Hematologic and genetic data of the probands with EPOR mutations.

\begin{tabular}{|c|c|c|c|c|c|c|c|c|c|c|}
\hline Case & Age & Sex & $\begin{array}{c}\mathrm{RBC} \\
\left(\times 10^{12} / \mathrm{L}\right)\end{array}$ & $\begin{array}{l}\text { Hemogiobin } \\
(\mathbf{H b}, \mathrm{g} / \mathrm{L})\end{array}$ & $\begin{array}{l}\text { Hematocrit } \\
\text { (Hct, \%) }\end{array}$ & $\begin{array}{l}\text { MCV } \\
\text { (iL) }\end{array}$ & $\begin{array}{c}\text { EPO } \\
\text { (UI/L) }\end{array}$ & $\begin{array}{l}\text { Mutation } \\
\text { cDNA }\end{array}$ & $\begin{array}{l}\text { Mutation } \\
\text { protein }\end{array}$ & $\begin{array}{l}\text { Family } \\
\text { History }\end{array}$ \\
\hline 1 & 52 & $\mathrm{~F}$ & 5.23 & 172 & $50 \%$ & & 1 & c.1142_1143delCC & p.Pro381Glnfs*2 & $\begin{array}{c}\text { Yes } \\
\text { (father and brother) }\end{array}$ \\
\hline 2 & 14 & $\mathrm{~F}$ & 7.1 & 200 & & 86 & $<1.0$ & c. $1220 \mathrm{C}>\mathrm{A}$ & p.Ser $407^{*}$ & $\begin{array}{c}\text { Yes } \\
\text { (mother and sister) }\end{array}$ \\
\hline 3 & 31 & M & 6.44 & 216 & 61.3 & & $<10.5$ & c.1293del & p.Ser432Alafs*21 & Yes (unknown) \\
\hline 4 & 8 & M & 7,3 & 197 & 57,5 & 78 & $<0,6$ & c.1293del & p.Ser432Alafs*21 & Yes \\
\hline
\end{tabular}


Table 2. Complications and treatment of the probands with EPOR mutations.

\begin{tabular}{lll} 
Case & Vascular complication & Phlebotomy \\
1 & Yes (myocardial infarction and transient ischemic attack) & Yes (twice/year) \\
2 & No & Yes \\
\hline 3 & No & No \\
4 & No & No \\
\hline
\end{tabular}

erythrocytosis at the age of 13 years. Her $\mathrm{Hb}$ was $172 \mathrm{~g} / \mathrm{L}$ when the NGS was performed. She also had a family history of idiopathic erythrocytosis (mother and sister), but the cause was unknown.

These two EPOR mutations have already been descri$\operatorname{bed}^{9,10}$ and are characterized as pathogenic.

The third mutation is a new frameshift mutation (c.1293del, p.Ser432Alafs*21) that was found in patient \#3: a 31-year old male native of New Caledonia now living in continental France. This result was confirmed by Sanger sequencing performed both on DNA extracted from an additional blood sample and from hair follicles in order to ensure the mutation was constitutional. $\mathrm{He}$ had been diagnosed with isolated idiopathic erythrocytosis at the age of 20 years. At the time of analysis, he was not showing any sign of splenomegaly nor any other medical condition. Due to his relocation in Europe and estrangement from his family, no data were available regarding a possible family history of erythrocytosis.

Since this mutation had not been previously reported in the literature or in databases, we enquired as to whether colleagues involved in EPOR sequencing had found a similar variant. Interestingly, this $E P O R$ variant had indeed been discovered by another French laboratory. Three patients were identified. An 8-year old boy (patient \#4) had been referred for isolated erythrocytosis in a context of a family history of idiopathic erythrocytosis in his mother, maternal aunt and uncle, and maternal grandfather who all presented isolated erythrocytosis that had not been previously investigated (Figure 1). The same laboratory had also found this mutation in a 48-year old female with isolated erythrocytosis, and a 54- year old female, also presenting with isolated erythrocytosis. For the second case (48-year old female), investigation of her family history revealed that her niece also carried the mutation. Both cases originated from New Caledonia, but further investigation found no relation between their families. However, the fact that the same, rare variant was identified independently in four patients from New Caledonia strongly supports a founder effect.

Since the discovery of the link between mutations in the gene encoding EPOR and erythrocytosis, ${ }^{11}$ many variants have been described. All are localized in exon 8 , which encodes the distal region of the EPO receptor. ${ }^{12}$ Most of the mutations cause a truncation in the intracellular domain of the receptor. It has now been established that this shortening leads to a loss of internalization and downregulation of the receptor, mostly due to the impairment of negative regulators binding to EPOR. Indeed, SHP1, which is a major inhibitor, binds to the residue p.Tyr454, and another inhibitor, SOCS3, to p.Tyr426, p.Tyr454, and p.Tyr456, all of which are lost in the various truncations described thus far. This results in an excessive activation of the receptor, showing hypersensitivity to EPO, combined with an increased number of receptors at the membrane, eventually leading to erythrocytosis. ${ }^{13-15}$ Nonetheless, recent studies have shown that

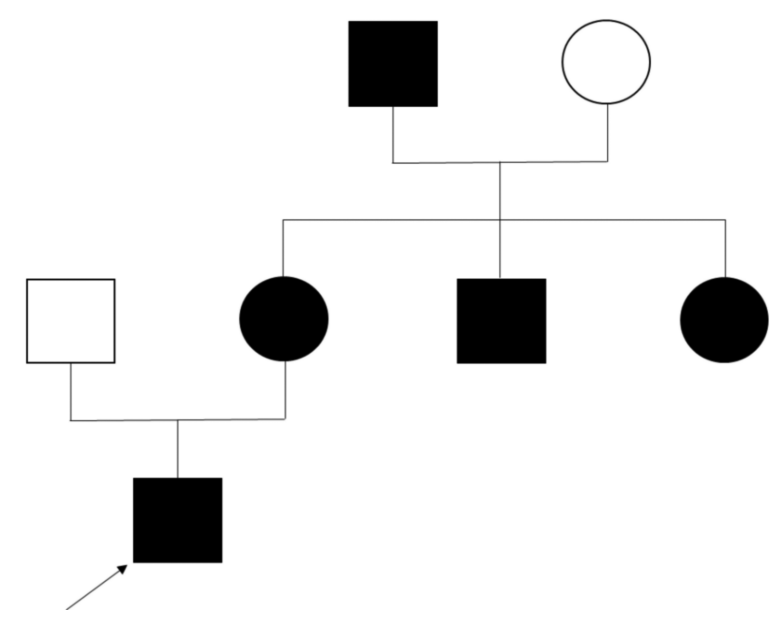

Figure 1. Pedigree of patient \#4. The propositus is indicated by the arrow. The black circles and squares represent people in the family with erythrocytosis.

the precise mechanisms leading to primary erythrocytosis vary depending on the type of the mutation, ${ }^{16}$ explaining the wide heterogeneity and complexity of this condition. Indeed, the mutant generates a new C-terminal cytoplasmic part that could induce the EPOR signaling pathway, as demonstrated in a recent study. ${ }^{16}$

The new variant described here (c.1293del, p.Ser432Alafs*21) is localized in a hot spot area where many pathogenic mutations have previously been reported. ${ }^{13,16-18}$ Due to its localization and the resulting truncation, it is expected to cause loss of the major binding sites of negative regulators, thus explaining the observed phenotype. The discovery of the same variant in three other families, for a total of four different families all coming from New Caledonia, with a history of erythrocytosis strengthens the hypothesis of a functional impact of the variant.

Regarding complications, only patient \#1 had a vascular complication as myocardial infarction and transient ischemic attack. On the contrary, neither hemorrhage nor progression to hematologic malignancies was noted in patients with EPOR mutations (Table 2).

In the whole cohort of patients with erythrocytosis, EPO values ranged from 0.6 to $140 \mathrm{IU} / \mathrm{L}$, with the vast majority of cases $(>90 \%)$ having a serum EPO in the normal range (4.3-29 UI/L). Off note, patients with an EPOR mutation all had low serum EPO, which is a classic feature of EPOR mutations.

In conclusion, our study confirms that mutations in $E P O-R$ are relatively rare among patients with idiopathic erythrocytosis. However, patients with low serum EPO levels and/or a family history of erythrocytosis should be checked for this anomaly. We also describe a novel mutation with a founder effect in New Caledonia. 
Mathilde Filser, ${ }^{1}$ Bernard Aral, ${ }^{2}$ Fabrice Airaud, ${ }^{3}$ Aurélie Chauveau, ${ }^{4}$ Aisha Bruce, ${ }^{5}$ Yann Polfrit, ${ }^{6}$ Anne Thiebaut, ${ }^{7}$ Martin Gauthier, ${ }^{8}$ Cédric Le Maréchal, 910 Eric Lippert, 4,9 Stéphane Béziau, ${ }^{3,11}$ Céline Garrec, ${ }^{3}$ Betty Gardie $e^{11,12,13^{*}}$ and François Girodon ${ }^{1,13,14^{*}}$

'Service d'Hématologie Biologique, Pôle Biologie, CHU de Dijon, France; 'Laboratoire de Génétique Chromosomique et Moléculaire, Pôle Biologie, CHU de Dijon, France; ${ }^{3}$ Service de Génétique Médicale, CHU de Nantes, Nantes, France; ${ }^{4}$ Hématologie Biologique, CHU de Brest, Brest, France; ${ }^{5}$ Department of Pediatrics, University of Alberta, Edmonton and Stollery Children's Hospital, Alberta Health Services, Alberta, Canada; 'Service de Pédiatrie, CH NOUMEA, Nouméa, Nouvelle-Calédonie; ' Service d'Hématologie, CHU Grenoble Alpes, Grenoble, France; ${ }^{8}$ Service d'Hématologie, Toulouse-Oncopole University Cancer Institute (IUCT-O), Toulouse, France; ${ }^{9}$ Université de Brest, Inserm, EFS, UMR 1078, GGB, Brest, France; ${ }^{10}$ Laboratoire de Génétique Moléculaire et d'Histocompatibilité, CHU de Brest, Brest, France; "L'Institut du Thorax, INSERM, CNRS, UNIV Nantes, Nantes, France; ${ }^{12}$ Ecole Pratique des Hautes, EPHE, PSL Research University, Paris, France; ${ }^{13}$ Laboratory of Excellence GR-Ex, France and ${ }^{14}$ Inserm U1231, Université de Bourgogne, Dijon, France

${ }^{*} B G$ and $F G$ contributed equally to this work as co-senior authors.

Correspondence:

FRANÇOIS GIRODON - francois.girodon@chu-dijon.fr

\section{doi:10.3324/haematol.2019.244160}

Disclosures: no conflicts of interests to disclose.

Contributions: MF and FG wrote the manuscript; $A B, Y P, A T, M G$ provided samples; $B A, A C, F A, E L, C L M$ and $C G$ performed analyses; $S B$ and $B G$ revised the manuscript.

Funding: this study was supported by grants from the ANR (PRTS 2015 "GenRED"); the labex GR-Ex, reference ANR-11-LABX-0051.

\section{References}

1. Pearson TC, Messinezy M, Westwood N, et al. A polycythemia vera update: diagnosis, pathobiology, and treatment. Hematology Am Soc Hematol Educ Program. 2000:51-68.

2. Barbui T, Thiele J, Gisslinger H, et al. The 2016 WHO classification and diagnostic criteria for myeloproliferative neoplasms: document summary and in-depth discussion. Blood Cancer J. 2018;8(2):15.

3. McMullin MF. Idiopathic erythrocytosis: a disappearing entity. Hematology Am Soc Hematol Educ Program. 2009:629-635.
4. Lappin TR, Lee FS. Update on mutations in the HIF: EPO pathway and their role in erythrocytosis. Blood Rev. 2019;37:100590.

5. Lenglet M, Robriquet F, Schwarz K, et al. Identification of a new VHL exon and complex splicing alterations in familial erythrocytosis or von Hippel-Lindau disease. Blood 2018;132(5):469-483.

6. Rumi E, Passamonti F, Pagano L, et al. Blood p50 evaluation enhances diagnostic definition of isolated erythrocytosis. J Intern Med. 2009;265(2):266-274.

7. Oliveira JL, Coon LM, Frederick LA, et al. Genotype-phenotype correlation of hereditary erythrocytosis mutations, a single center experience. Am J Hematol. 2018;93(8):1029-1041.

8. Girodon F, Airaud F, Garrec C, Bézieau S, Gardie B. Gene panel sequencing in idiopathic erythrocytosis. Haematologica. 2017; 102(1):e30.

9. Al-Sheikh M, Mazurier E, Gardie B, et al. A study of 36 unrelated cases with pure erythrocytosis revealed three new mutations in the erythropoietin receptor gene. Haematologica. 2008;93(7):10721075.

10. Toriumi N, Kaneda M, Hatakeyama N, et al. A case of primary familial congenital polycythemia with a novel EPOR mutation: possible spontaneous remission/alleviation by menstrual bleeding. Int J Hematol. 2018;108(3):339-343

11. de la Chapelle A, Traskelin AL, Juvonen E. Truncated erythropoietin receptor causes dominantly inherited benign human erythrocytosis. Proc Natl Acad Sci U S A. 1993;90(10):4495-4499.

12. Chauveau A, Luque Paz D, Lecucq L, et al. A new point mutation in EPOR inducing a short deletion in congenital erythrocytosis. $\mathrm{Br}$ ] Haematol. 2016;172(3):475-477.

13. Sokol L, Luhovy M, Guan Y, Prchal JF, Semenza GL, Prchal JT. Primary familial polycythemia: a frameshift mutation in the erythropoietin receptor gene and increased sensitivity of erythroid progenitors in erythropoietin. Blood. 1995;86(1):15-22.

14. Perrotta S, Cucciolla V, Ferraro M, et al. EPO receptor gain-of-function causes hereditary polycythemia, alters CD34+ cell differentiation and increases circulating endothelial precursors. PLoS One. 2010;5(8):e12015.

15. Watowich SS, Xie X, Klingmuller U, et al. Erythropoietin receptor mutations associated with familial erythrocytosis cause hypersensitivity to erythropoietin in the heterozygous state. Blood. 1999:94(7):2530-2532.

16. Pasquier F, Marty C, Balligand T, et al. New pathogenic mechanisms induced by germline erythropoietin receptor mutations in primary erythrocytosis. Haematologica. 2018;103(4):575-586.

17. Furukawa T, Narita M, Sakaue M, et al. Primary familial polycythaemia associated with a novel point mutation in the erythropoietin receptor. Br J Haematol. 1997;99(1):222-227.

18. Bento C, Percy MJ, Gardie B, et al. Genetic basis of congenital erythrocytosis: mutation update and online databases. Hum Mutat. 2014;35(1):15-26. 